\title{
Superhydrophobic Copper Tubes with Possible Flow Enhancement and Drag Reduction
}

\author{
Neil J. Shirtcliffe, Glen McHale, ${ }^{*}$ Michael I. Newton, and Yong Zhang
}

School of Science and Technology, Nottingham Trent University, Clifton Lane, Nottingham NG1 1 8NS, U.K.

\begin{abstract}
The transport of a Newtonian liquid through a smooth pipe or tube is dominated by the frictional drag on the liquid against the walls. The resistance to flow against a solid can, however, be reduced by introducing a layer of gas at or near the boundary between the solid and liquid. This can occur by the vaporization of liquid at a surface at a temperature above the Leidenfrost point, by a cushion of air (e.g. below a hovercraft), or by producing bubbles at the interface. These methods require a continuous energy input, but a more recent discovery is the possibility of using a superhydrophobic surface. Most reported research uses small sections of lithographically patterned surfaces and rarely considers pressure differences or varying flow rates. In this work we present a method for creating a uniform superhydrophobic nanoribbon layer on the inside of round copper tubes of millimetric internal radius. Two types of experiments are described, with the first involving a simultaneous comparison of four tubes with different surface finishes (as received, as received with hydrophobic coating, nanoribbon, and nanoribbon with a hydrophobic coating) under constant flow rate conditions using water and water-glycerol mixtures. The results show that the superhydrophobic nanoribbon with a hydrophobic coating surface finish allows greater flow at low pressure differences but that the effect disappears as the pressure at the inlet of the tube is increased. The second experiment is a simple visual demonstration of the low-pressure behavior using two nominally identical tubes in terms of length and cross-section, but with one tube possessing a superhydrophobic internal surface finish. In this experiment a reservoir is allowed to feed the two tubes with open ends via a T-piece and it is observed that, once flow commences, it preferentially occurs down the superhydrophobic tube.
\end{abstract}

KEYWORDS: superhydrophobic $\bullet$ liquid flow $\bullet$ tubes $\bullet$ slip $\bullet$ friction $\bullet$ plastron

\section{INTRODUCTION}

T 1756 , Leidenfrost observed that a droplet of water deposited on a hot surface does not instantly vaporize but remains as a droplet for a considerable duration of time and slides freely across the solid surface (1). This is because a layer of water at the solid-water interface vaporizes and forms a cushion of air (2). The first scientific descriptions of possible air-cushioned vehicles arose a century ago (3). By the 1950s, a technical solution to creating a layer of air between the hull of a boat and the surface of water to reduce drag had been found with the invention of the hovercraft (4). In 1973, U.S. naval engineers dragged a cylinder coated with bubbles generated by electrolysis through water and showed a reduction in skin friction (5). This led on to the creation of slippery surfaces for ships through the active release of microbubbles underneath the hull (6). In this approach, a continuous input of energy is needed, the bubbles are dynamic and are not fixed around the surface of the solid, and, as they detach from the surface, turbulence is created. In contrast, some aquatic insects have body structures which passively fix and link microbubbles to create and retain a film of air (a plastron) when they are submerged; this structure is used to allow underwater

* To whom correspondence should be addressed. E-mail: glen.mchale@ ntu.ac.uk.

Received for review March 21, 2009 and accepted May 18, 2009

DOI: 10.1021 /am9001937

๑ 2009 American Chemical Society breathing without the need for gills (7). Their surfaces use rigid hydrophobic hairs, creating a superhydrophobic surface, so that surface tension pins the water and prevents it penetrating between the hairs.

In recent years, many methods of producing super waterrepellent (superhydrophobic) surfaces using a combination of chemical functionality and small-scale topography have been developed (for reviews see refs 8 and 9), and it has been shown that these can mimic plastrons, the trapped gas layers that some aquatic insects use to breathe (10). It has been speculated that drag may be reduced when water flows across superhydrophobic surfaces $(11-22)$ and results from plates and microfluidic channels suggest some drag reduction corresponding to micrometer-size slip lengths might exist (12-22). However, these systems almost all involve modification of an easily accessible surface and have been limited mainly to microchannels and closely spaced plates. These results have been a matter of some controversy due to the potential for large margins of uncertainty in the experimental data $(17,23)$. However, a recent study carried out in the absence of external pressure on a flat, microlithographically patterned surface showed a very large effect, which the authors suggested indicated a slip length of several hundred micrometers (24). Most recently, we have shown that a large (several centimeter diameter) spherical solid object can achieve a higher terminal velocity in water when it possesses a superhydrophobic surface which retains a film of air when submerged (i.e. a plastron) (25). In that work, it 


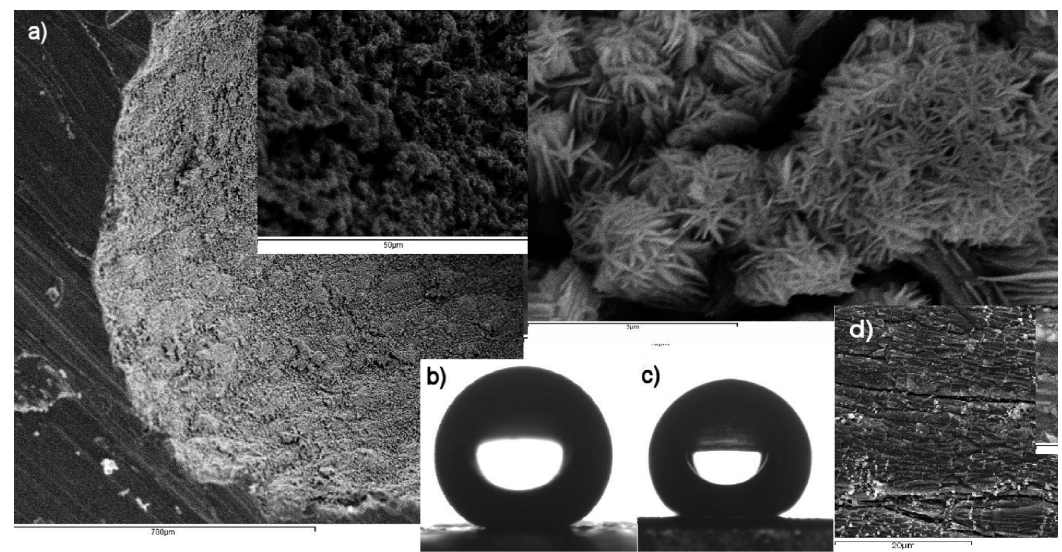

FIGURE 1. (a) Electron microscope images of hydrophobic nanoribbon decorated internal copper surfaces of tubes. Side-profile optical images of droplets of (b) water, and (c) glycerol on the surface shown in (a). The original surface is shown in (d).

was noted that bubbles of gas with nonrigid vapor-liquid interfaces are known to have large reductions in drag compared to an equivalent solid object, due to the ability of the internal gas to circulate within the bubble (so-called Hadamard-Rybczinski drag) (26-29). In such a view, continuity of stress across the gas-liquid interface is the fundamental boundary condition rather than a no-slip or slip boundary condition.

In this report, we first describe a simple coating process that allows the difficult-to-access internal surfaces of circular tubes of copper to be converted to superhydrophobic. This process involves a step to create a copper nanoribbon decorated surface followed by a subsequent hydrophobic treatment, so that four types of internal surface finish (as received, hydrophobic as received, copper nanoribbon decorated as received, and hydrophobic copper nanoribbon decorated as received surfaces) may be studied. The only difference between tubes, which are of nominally identical cross-section and length, is the internal surface finish. We then describe an experiment involving simultaneous measurements of pressure differences for constant flow rate of water and $50 \% \mathrm{w} / \mathrm{w}$ water-glycerol solutions through four tubes with these different internal surface finishes. These results show that a lower pressure drop along a tube can be observed when the internal surface finish is superhydrophobic, provided the internal pressure is low. Motivated by the implied difference in flow rates, we then present a visualization experiment showing how a tube with an internal superhydrophobic surface presents a flow path preferential to one whose internal surface finish is not superhydrophobic. The observed effect is valid at the macroscale and agrees with the hydrofoil test of Gogte et al. (18), suggesting that technically useful results are indeed possible.

\section{EXPERIMENTAL SECTION}

Superhydrophobic Coatings. At the beginning of this work, we anticipated that measurements of differences in flow rates through tubes with millimetric inside diameters due to different internal surface finishes could provide many sources of systematic error. The experiment was therefore designed so that a set of four geometrically identical tubes (i.e. possessing the same internal diameter, length, and shape and originating from the same base stock) could be simultaneously tested with tube position in the flow system being randomized in each experiment. In choosing our materials approach, we specifically avoided any tube production method that would involve seams or joins along the length of the channel or tube. We also sought a method to transform the internal surfaces without creating any significant change in tube internal radius and which would be able to produce a uniform coating along the full length of a tube. A final condition was that we needed a method that could produce a rough surface that could subsequently be transformed to superhydrophobic by a change of the surface chemistry, so that direct comparisons of the effect of a change of hydrophobicity alone could be tested.

The approach we adopted to creating samples was to use as the base system commercially available half-hard copper tubing (Industrial Ancillaries U.K.) that were relatively stiff and supplied straight. To alter the internal surfaces of the copper tubes, we significantly adapted a system previously developed for generating rough copper oxide nanoribbons on flat copper plates (30). When hydrophobized, these surfaces produce an extremely water-repellent surface with contact angles to water of $160^{\circ}$ and low contact angle hysteresis indicating the Cassie state. In the tube case, tubes of internal radius $r=0.876 \mathrm{~mm}$ were precut by hand and then affixed to a sheet of glass parallel to one another with wax before being cut to length with a wafer dicing saw. Experiments were conducted with tubes of length $l=110$ $\mathrm{mm}$ or $l=84 \mathrm{~mm}$ with all four tubes in a given experiment cut to the same length. Although the absolute lengths differed slightly from one set of four tubes to another, the lengths of the individual tubes within any given set of four were the same to within an accuracy of $0.005 \mathrm{~mm}$, according to digital caliper measurements. After the ends were reamed with a diamondcoated conical bit, the lengths were dewaxed and degreased by Soxhlet extraction using diethyl ether, toluene, and then ethanol (Haymond U.K.). Each stage was carried out for $4 \mathrm{~h}$ before the tube was allowed to dry. Half of the tube sections were then connected to a peristaltic pump, and $36 \mathrm{mM}$ ammonia solution diluted from stock ( $35 \%$ Fischer, U.K.) was circulated in them at $5{ }^{\circ} \mathrm{C}$ in a cold room. The tube order on the pump and flow direction were rotated every 2 days for 10 days. This process was deliberately chosen to ensure the concentration gradient along the tubes was minimized and kept constant, although this did result in a lengthy process time. The process generates copper hydroxide nanoribbons aligned with the long axis perpendicular to the surface. Rinsing the tubes with deionized water and then ethanol, drying, and then heating to $180^{\circ} \mathrm{C}$ for $2 \mathrm{~h}$ heating at $0.1^{\circ} \mathrm{C} \mathrm{min}{ }^{-1}$ before allowing them to cool slowly in a Carbolite furnace converts the copper hydroxide to copper oxide, which can then be easily rendered hydrophobic. 
Scanning electron micrographs (SEM) of sections of tubes are shown in Figure 1. The observed nanoribbons were found to be around $1 \mu \mathrm{m} \times 100 \mathrm{~nm} \times 6 \mathrm{~nm}$ in size and aligned with the long axis perpendicular to the surface, similar to the results for the flat surface (higher resolution TEM pictures of nanoribbons on flat surfaces are also shown in Figure 1). The original surface of the copper tubes (referred to here as "as received") prior to creating the nanoribbons is shown in Figure $1 \mathrm{~d}$ and displays a crystalline microstructure with a roughness on a typical length scale of tens of micrometers. Thus, the nanoribbon decorated surfaces are comprised of a micrometer length scale roughness overlaid by the nanometer-scale roughness of the treatment, thus generating a multi-scale surface. A selection of the original tube sections and the nanoribbon decorated tube sections were coated with a commercial fabric waterproofing agent (Grangers' U.K. Extreme wash-in solution) to render them superhydrophobic; this process was also applied to flat plates for comparison. Grangers' Extreme wash-in is a proprietary formula, but similar coatings are known to be water emulsions of a single component, a perfluoroalkoxy polymer with the alkoxy groups being vinyl esters or acrylic. On heating, the hydrophilic groups release water, turn in, and cross-link, presenting a perfluorocarbon surface to the environment. The solution was diluted $1: 20$ in deionized water and washed through the tubes for 10 min. They were then thoroughly rinsed with deionized water before being air-dried and then placed in an oven with controlled convection and heated to $100{ }^{\circ} \mathrm{C}$ for $2 \mathrm{~h}$. In our experiments, surfaces treated with this waterproofing agent showed contact angles to both water and organic compounds very close to those of PTFE. These processes allowed three types of internal surface finishes to be created from the as received tubes. The first was a hydrophobic surface, the second was a nanoribbon surface, and the third was a hydrophobic nanoribbon surface. Thus, four types of internal finish, created from a single original tube, were available for comparison between the effect of inherent surface roughness and the nanoribbon decorated surface and both of these with a hydrophobic surface chemistry.

Constant Flow-Rate System with Four Simultaneous Tubes. The first type of experiment performed was the simultaneous measurement of pressure drops along four tubes during an imposed constant flow rate with either water or $50 \% \mathrm{w} / \mathrm{w}$ water-glycerol mixtures. To avoid anticipated experimental errors related to comparing data from separate experiments on individual tubes, the experimental system used four parallel arms with the four types of tubes randomly switched between positions to eliminate systematic errors (Figure 2). The constant flow rate was provided by a high-force (66 lb average linear force) syringe pump (Harvard PHD22/2000, U.S.) connected to four 50 or $100 \mathrm{~mL}$ glass syringes with matched barrels (Popper, U.S.) designed to eliminate leakage at high pressures. The syringes were attached using short sections of silicone tubing to polypropylene T-pieces, and the through port of the T-piece was connected via silicone tubing to the test sections of the copper tube. The two flexible sections of tubing allowed the test sections to be leveled and aligned by fixing them to a flat plate, thus minimizing effects from height differences. The third opening of each T-piece was connected to a pressure sensor (26PC 1 PSI, Honeywell, U.K.) pointing upwards, which allowed the pressure sensors to be used dry. The outlets of the tubes were mounted back from the edge of the mounting plate to prevent liquid droplets from forming at the ends of the tubes. In preliminary experiments it was found that without this arrangement the accuracy and sensitivity of the system was such that a pulsating back-pressure due to droplet formation and detachment was problematic. The preliminary measurements also considered an arrangement using brass three-way taps and $15 \mathrm{~mm}$ diameter copper plumbing tees with the sensors wetted and mounted downwards; consistent results were also achieved. a)
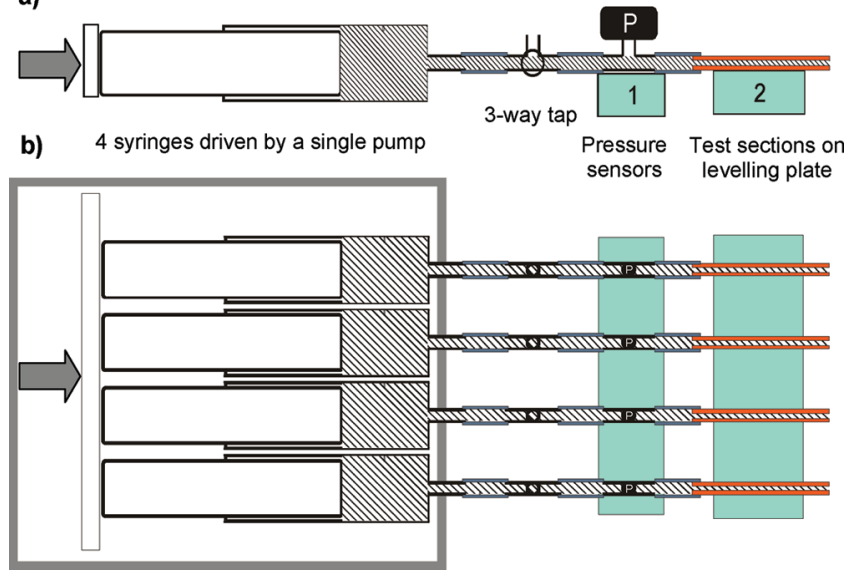

FIGURE 2. Diagram of constant flow method using four glass syringes connected to four copper tubes with differing internal surface finishes and allowing simultaneous measurement of pressure drop across each tube: (a) side view; (b) top view. The four glass syringes were driven by a single high-force syringe pump, and the pressure sensors were connected by flexible tubes to allow common alignment; test sections were mounted on a common leveling plate to minimize pressure offsets and control flow.

The pressure sensors used were the 26PC series 100 mbar or 1 psi calibrated, compensated, differential, piezoresistive sensors (Sensortechnics and RS, U.K.). The sensors were powered at 10 $\mathrm{V}$ dc using a smoothed power supply, and the sensor voltage was read at $2.3 \mathrm{~s}$ intervals into a digital voltmeter (Keithley $175 \mathrm{~A})$ and saved onto a computer using LabView software and a GPIB interface card.

Experiments were carried out by randomly connecting the pressure sensors and the copper tubes using silicone tubing. Deionized water and glycerol (Fisher U.K., >99.0\%) were mixed as test solutions, thus allowing the viscosity to be increased from $1.005 \mathrm{mPa} \mathrm{s}$ (at $20^{\circ} \mathrm{C}$ ) by around a factor of 6 for $50 \% \mathrm{w} / \mathrm{w}$ water-glycerol mixtures. Tube clamps were used to enhance the connections and prevent leakage, and the syringes were then connected and filled via the three-way taps. Liquids were then allowed to equilibrate to allow gas bubbles formed during filling to redissolve or disperse. The time allowed for this was found to depend on the solution used, but with water $20 \mathrm{~min}$ was sufficient and for $50 \%$ glycerol this extended to $30 \mathrm{~min}$; waiting for a longer time had no measurable effect on the results. Measurements were then taken by setting the syringe pump to start at a low flow rate $\left(2 \mathrm{~cm}^{3} \mathrm{~min}^{-1}\right)$ and to pump for 5 min before shutting it off. Pressure measurements were taken as an average of the measurements made during the stable plateau of pressure, ensuring that a steady state had been reached. Measurements were made over a range of flow rates for each test system using increasing flow rates. This ensured a single series of flow-rate measurements could be made for all four copper tubes with their different internal surface finishes with no change to any connections within the system. While this has clear advantages in minimizing errors in any one sequence, the fixed volumes available in the syringes means that the method did limit the number of different flow rates that were possible in a single experiment. The data were analyzed by selecting a region where the pressure was constant and averaging (typically 20 measurements) the sensor response over this period. Sets of data for reducing flow rates were also taken, and these were almost identical with the rising pressure results. By repeating experiments with the different pipes switched between positions, it was confirmed that the observed data were not due to any differences in the syringes or pressure sensors.

Visualization Experiments. In the design of the initial type of experiment, it had been unclear how large any drag reduction 


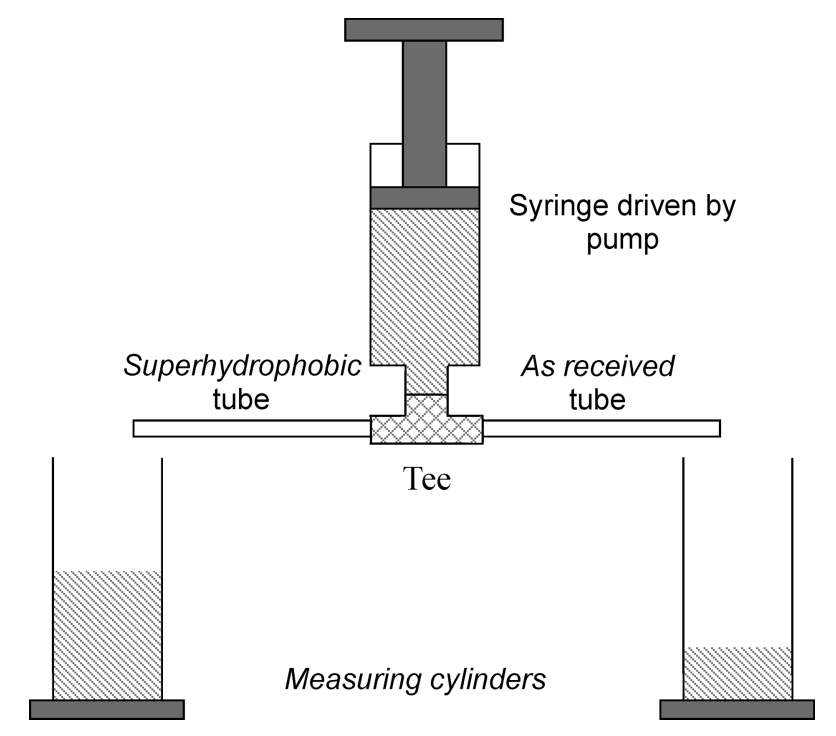

FIGURE 3. Diagram of visualization experiment using two copper tubes with differing internal surface finishes (one as received and one hydrophobic copper nanoribbon decorated).

effect might be or whether such an effect might be observed at all. However, data from the experiments indicated a sufficiently large difference when a copper tube had a superhydrophobic internal surface that a complementary visualization experiment was designed. In this second type of experiment, the syringe pump was used to force water from a single syringe via a T-piece into two horizontally mounted copper tubes of identical radii and length (Figure 3). The outflow was then collected in two measuring cylinders so that the flow rates could be directly compared using the same liquid at the same temperature and pressure for each. To initiate flow down both tubes when one had a superhydrophobic internal finish, the ends were momentarily blocked and then released. This simple experiment was used to provide videos showing the effect at different flow rates of tubes with different surface finishes (available in the Supporting Information).

\section{RESULTS}

When the pressure drops along a tube as a function of flow rate for both water and $50 \%$ w/w water-glycerol mixtures were measured for the as received tubes, the behavior was found to follow a linear Poiseuille relationship, $\dot{Q}_{0}=\pi r^{4} \Delta p / 8 \eta l$, where $\eta$ is the viscosity of the fluid, $\Delta p$ is the pressure drop between the ends of the tube, and $r$ and $l$ are the internal radius and length of the tube, respectively. This remained true when the copper nanoribbon internal surface finish was created. This indicates that the process of creating copper nanoribbons alone does not result in any observable effect due to a change in internal radius or the change in topography of the surface. The flow regime at these flow rates in tubes of this size is in the laminar region with a Reynolds number, Re, of a maximum of 850 for a highest flow rate of $70 \mathrm{~mL} \mathrm{~min}^{-1}$ for water. For $50 \% \mathrm{w} / \mathrm{w}$ water-glycerol mixtures with a flow rate of $26 \mathrm{~mL} \mathrm{~min}^{-1}$, the Reynolds number is far lower at $R e=59$. In both cases, the flow patterns are expected to be laminar, and this is consistent with the effect of the roughness of the walls not being expected to be important.

The effects on the pressure drops along each of the three tubes of length $l=84 \mathrm{~mm}$ possessing hydrophobic, nanor-
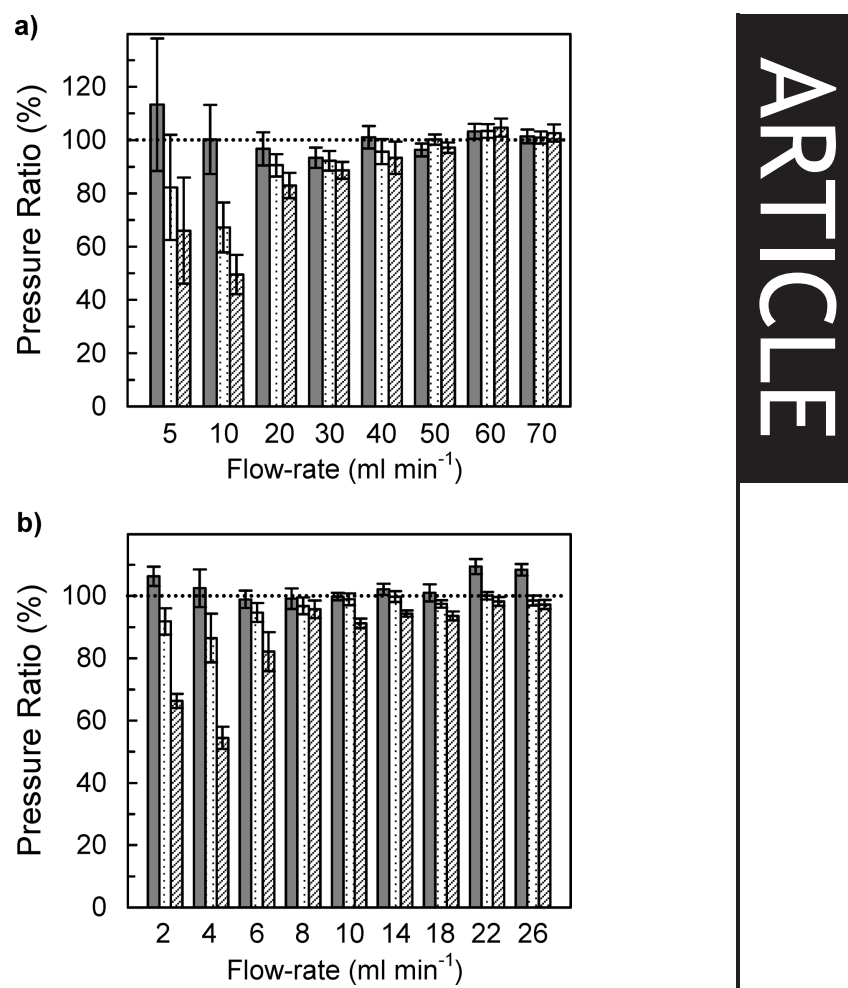

FIGURE 4. Ratio of pressure drop (as a percentage) along tubes with different internal surface finishes compared to the as received tubes at various flow rates for (a) water and (b) $50 \% \mathrm{w} / \mathrm{w}$ water-glycerol. Each group of three bars shows tubes with three internal surface finishes: copper nanoribbon decorated (shaded bar), hydrophobic as received (dotted bar), and hydrophobic copper nanoribbon decorated (diagonal filled bar). Error bars are from the variation in pressure readings during a measurement sequence at a given constant flow rate.

ibbon decorated, and hydrophobic nanoribbon decorated internal surfaces compared to the as received tube (taken as the reference) are shown in Figure 4. The trends in Figure 4 are representative of repeat experiments conducted with sets of four copper tubes manufactured from new starting tubes (a total of two sets with $l=84 \mathrm{~mm}$ and three sets with $l=115 \mathrm{~mm}$ ). For both water and $50 \% \mathrm{w} / \mathrm{w}$ water-glycerol mixtures, the pressure drop along the copper nanoribbon decorated tube is close to that observed with the as received tube. Up to flow rates of $50 \mathrm{~mL} \mathrm{~min}^{-1}$ (corresponding to 3.8 mbar) and $8 \mathrm{~mL} \mathrm{~min}^{-1}$ (corresponding to $3 \mathrm{mbar}$ ) for water and the $50 \% \mathrm{w} / \mathrm{w}$ water-glycerol mixture, respectively, there appears to be an effect for the hydrophobic as received tube. These as received tubes do not have a perfectly smooth surface but have a micro-rough surface. However, the greatest effect is for the hydrophobic nanoribbon decorated tube, where the reduction in pressure drop in this lower flow-rate regime is far greater for both the water and the $50 \% \mathrm{w} / \mathrm{w}$ water-glycerol mixture. At these lower flow rates, the pressure drop is large and enters the range $50-70 \%$ of the values found for the as received tube; the error estimates suggest this is a significant difference. At higher flow rates, which correspond to higher internal pressures, the data return to values similar to those of the as received tubes.

The implication from Figure 4 is that a large flow rate difference between a superhydrophobic and an as received copper tube would occur if a constant pressure were applied 


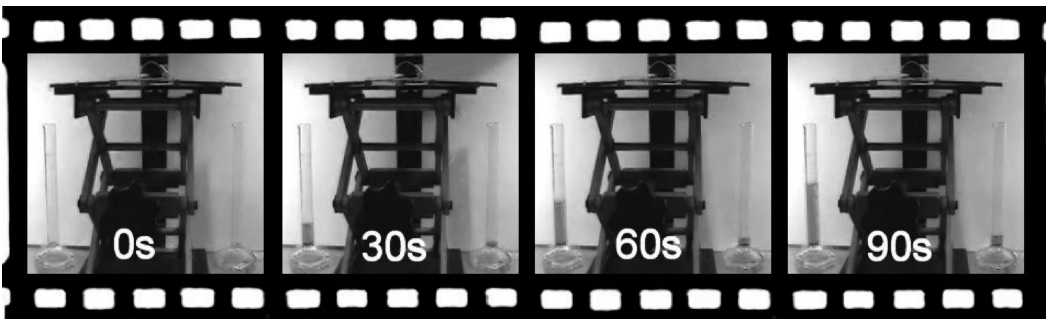

FIGURE 5. Sequence of images taken during flow of water at $5 \mathrm{~mL} \mathrm{~min}^{-1}$ through a superhydrophobic tube and an as received tube.

and the flow rate was in the lower flow-rate regime. The second type of experiment using two tubes connected to a single syringe pump was designed to confirm whether this was indeed the case. When two tubes with the same internal surface finishes were tested against each other, little difference in the volumes collected from their outlets was observed. However, when one tube had the superhydrophobic internal surface finish and the flow rate was in the lower pressure regime, the situation was different. The images in Figure 5 show a time sequence for a flow rate of $5 \mathrm{~mL} \mathrm{~min}^{-1}$ of water imposed by the syringe pump using two tubes from a set of four. It is clear that flow through the superhydrophobic tube is preferred, compared to the as received tube, and a visibly larger volume is collected from its outlet. In this experiment the outlets of both tubes had to be momentarily blocked using a finger tip to initiate flow down the tubes; otherwise, the water would not initially enter the superhydrophobic tube. Experiments were carried out for the same range of flow rates as in Figure 4, and preferential flow down the superhydrophobic tube was observed up to the threshold flow rates corresponding to higher pressure within the tube. This was true whichever of the two arms was used for the superhydrophobic tube. We found that these effects were reproducible and provided clear visual indicators of differences in flow rates for tubes whose only difference was the internal surface finishes.

\section{DISCUSSION}

Accuracy and Errors. Claims of differences in drag for flow across superhydrophobic surfaces have been a matter of controversy in the literature, and a careful consideration of possible errors is therefore important. In any flow-rate measurement, temperature and pressure errors are potential sources of inaccuracy. In our first type of experiment, measurements of flow through four tubes with the different internal surface finishes are performed simultaneously, thus minimizing the risk of differences in the liquid viscosity and other properties. Similarly, any comparison between the four surface finishes for a given flow rate should not involve large errors from temperature differences. In terms of the different flow rates that were investigated, any one experimental sequence completed a full set of flow rates without disturbing the apparatus (e.g. to refill the syringes). In addition, equipment and solutions were sited in temperature-controlled laboratories with control to $1^{\circ} \mathrm{C}$ over $24 \mathrm{~h}$ and with considerably lower temperature drift during the measurement period. Pressure readings from the tubes were taken as an average of 10-20 measurements during flow with base (zero flow) readings measured between flow measurements, and the deviation of the measurements was used to generate the error bars in Figure 4. Although these do not take into account systematic errors due to tube sizes or blockages, we think the errors due to these factors are relatively small, due to the fact that all five different sets of four tubes behaved in broadly the same manner, although the magnitude and onset of the effect varied slightly (probably due to variations in the quality of the copper nanoribbon coating), which is why the results have not been combined and only a representative data set has been presented in Figure 4. Pressure measurements were always carried out from a resting zero flow, thus ensuring hysteresis of the detectors was avoided by always approaching the set flow rate from zero. Calibration plots for the pressure sensors were almost linear to 500 mbar, which is twice the maximum pressure measured during flow; sensors were not taken close to their proof pressure of 1.4 bar. These sensors were used for their greater sensitivity and higher response at lower pressures. Two port sensors were used as differential sensors to allow for changes in atmospheric conditions; the second port on these sensors is more protected from accidental wetting than those configured only for differential sensing

Whether or not liquids are in equilibrium with uniform properties (homogenous mixing, presence of bubbles, etc.) could introduce inaccuracies. To minimize this risk, prior to a set of experiments the system was assembled and filled using a syringe, and then liquid was flowed through at a low rate for some time, with longer periods $(3 \mathrm{~h})$ being used for the glycerol-water mixtures to ensure the entire system was filled with the mixture desired. We considered whether wall demixing might occur for the $50 \% \mathrm{w} / \mathrm{w}$ water-glycerol mixtures when the surface finishes were hydrophobic and so lead to a region enriched in glycerol at the walls and one of water in the center; this would not be an issue for experiments with pure water. However, this would imply that in a steady-state flow the flow rate would be lower at a hydrophobic surface than at a hydrophilic one. Another possible error could be the presence of bubbles within tubes, but we believe it is unlikely this would result in apparent reduced drag for the tubes with hydrophobic surface finishes. Glycerol solutions far from equilibrium with the atmosphere can change viscosity as they absorb or release water. By taking into consideration our laboratory temperature of $20^{\circ} \mathrm{C}$ and relative humidity of $55 \%$, this risk was minimized by using $50 \% \mathrm{w} / \mathrm{w}$ glycerol-water and using a large volume.

Further sources of possible error are differences in the geometrical factors of the tube. The pressure difference 
along a tube, using Poiseuille's equation and assuming a negligible Bernoulli effect, is linearly dependent upon the length of the tubes. In preparing tubes for a given experiment, we cut them from the same initial long tube, by laying sections alongside each other and cutting both ends using a wafer dicing saw. The variation in length of the tubes was less than $20 \mu \mathrm{m}$, as measured with digital calipers. Once cut, burrs were removed by using a conical diamond bit to ream the ends of the pipe by hand, thus generating a 50-100 $\mu \mathrm{m}$ conical region. This minimized any effects due to change of radius, which has an $r^{4}$ dependence in Poiseuille's equation, but effectively increased the uncertainty in tube length to $120 \mu \mathrm{m}(\sim 0.1 \%)$, since the conical region extends the length over which the pressure changes.

It is possible that tubes may have had radius variations created during manufacture or due to bending. To reduce these effects, half-hard copper was used, which is delivered as straight lengths and is more difficult to bend than normal copper tubing. Sections were assigned randomly to each surface treatment to minimize systematic error, and results were consistent across eight sets of tubes cut from two stock sections ordered on separate occasions several months apart. A total of 24 tubes were used, as some were sets of 2 rather than 4 . We do not, therefore, believe the effects observed were due to a systematic radius error. In the different sets of experiments, some tubes with superhydrophobic surfaces appeared to be more effective than others, suggesting that roughness was not even between samples. We do not believe this is surprising, given the internal surface being modified was closed and inaccessible. Although data from the first type of experiment were not identical, they all behaved in the same way. A further complication is that after several runs in potentially corrosive glycerol-water mixtures the tubes' properties altered slowly.

Physical Interpretations. We believe that both of the types of experiments we have performed show that a significant reduction in drag and wall friction in tubes can be observed when the internal surfaces of the copper tubes are converted to superhydrophobic by the creation of hydrophobic copper nanoribbons and the flow does not induce pressures that are too high. We believe this is related to the flow of liquid across the surface with a Cassie state involving little contact with the solid surface.

A common approach to drag reduction data is to invoke a slip-boundary condition. When the speed of a liquid flowing across a static solid surface is non-zero at the solid-liquid interface, the slip velocity $\Delta v_{\mathrm{s}}$ can be related to the shear stress $\tau$ by $\Delta v_{\mathrm{s}},=\tau / \kappa$, where $\kappa$ is the solid-liquid coefficient of friction $(31,32)$. This can be converted to a slip length, $b=\eta / k$, giving the distance beyond the boundary that the flow profile would need to be extrapolated to reach zero velocity. The current case is equivalent to slip if the thickness of the air film is ignored. The flow rate is then described by a slip-corrected Poiseulle formula: $(33,34)$

$$
\dot{Q}=\dot{Q}_{0}\left(1+\frac{4 b}{r}\right)
$$

In the data from Figure 4, the slip length can be estimated from the percentage change in pressure compared to the as received tubes. For water at flow rates of 5, 10, 20, 30, and $40 \mathrm{~mL} \mathrm{~min}^{-1}$, the estimated slip lengths are $74 \pm 66,110 \pm$ $23,37 \pm 15,25 \pm 10$, and $20 \pm 14 \mu \mathrm{m}$, respectively; above these flow rates the error estimates on the slip lengths incorporate zero. Quantitative estimates of the experimental uncertainties are difficult, but these estimates are our maximum estimates. For the $50 \%$ w/w water-glycerol solutions and flow rates of $2,4,6,810,14$, and $18 \mathrm{~mL} \mathrm{~min}^{-1}$ the estimated slip lengths are $73 \pm 7,99 \pm 11,39 \pm 16,9 \pm 8$, $19 \pm 4,12 \pm 3$, and $14 \pm 4 \mu \mathrm{m}$, respectively. At higher flow rates slip lengths are close to zero and zero is within the error range. While the uncertainty on measurements is large in absolute terms, for the lower flow rates, the data are significantly above the zero value. Moreover, the largest of these slip lengths is of the same order of magnitude of the giant slip in the range $80-185 \mu \mathrm{m}$ recently reported by Lee et al. (24). In lithographically fabricated superhydrophobic systems with regular arrays of posts, it has been suggested that a slip length will be on the order of the feature period scaled by $\left(1-\varphi_{\mathrm{s}}\right)^{1 / 2}$, where $\varphi_{\mathrm{s}}$ is the Cassie solid fraction $(16,24)$. For disordered surfaces, provided by the type of process we have used, a criterion for large slip is that the Cassie solid fraction should be very small. While the Cassie solid fraction cannot be directly measured in our case, a strong indication of a small Cassie fraction is that the observed contact angles should be sufficiently close to $180^{\circ}$ so as to be effectively nonmeasurable due to the inability to stabilize a droplet on the surface. Direct visual measurement of contact angles on the inside of our millimetric diameter copper tubes was not possible, but it is known that the process we used can produce highly superhydrophobic surfaces on flat copper due to the dual-length scale structure (35). Previous studies on whether slip can exist when the internal walls of smooth capillaries are hydrophobic has been reviewed by Vinogradova, (33) and these reports would be instructive for extending our work to include measurements of advancing contact angles.

The concept of a slip length originates from consideration of fluid flow across flat surfaces. The slip length extrapolates the bulk flow profile a fixed distance beyond the solid-fluid boundary to where the velocity would vanish. A superhydrophobic surface is topographically structured, and the Cassie state uses this structure to allow the liquid to bridge across surface asperities so that the liquid does not retain intimate contact with the solid surface. In this context, the application of the slip length concept to data, such as Figure 4 , is quite different to a slip length at the solid-liquid interface of a smooth solid or even a rough solid on which the liquid retains contact at all points. The fundamental boundary condition between two phases is the continuity of shear stress. When one of those phases is a solid and the other is a liquid, the equivalent statement is that a no-slip boundary condition exists. However, when the two phases are a gas and a liquid, this is no longer the case. A wellknown example is the terminal rise velocity of a slowly rising 
a)

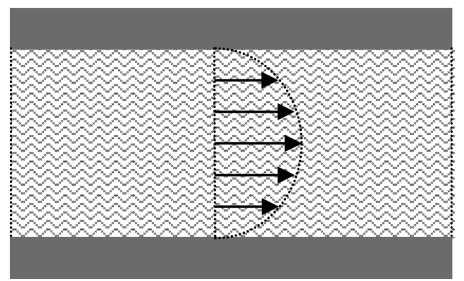

b)

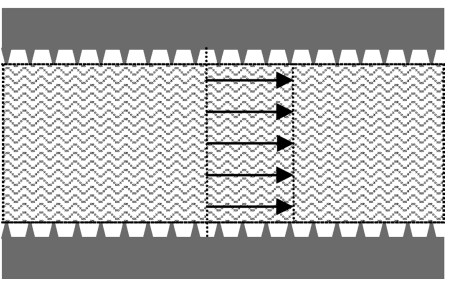

FIGURE 6. Schematics of flow patterns: (a) Poiseuille flow in a closed channel with no-slip boundary conditions; (b) plug flow with a sheathing film of air generated by hydrophobic nanodecorations on the channel walls.

gas bubble. In such a situation, the friction from the external liquid induces an internal circulation of the gas and this reduces the drag (known as Hadamard-Rybczinski drag) by $33 \%$ compared to the Stokes drag on an equivalent solid sphere (26-29). When experiments of this type are performed, these two types of drag can be directly observed for gas bubbles with and without rigidified gas-liquid interfaces. Thus, we believe, it is possible to consider drag reduction for flow through a superhydrophobic tube in similar terms (25). Since the Cassie fraction for a superhydrophobic surface can be extremely small, the liquid may effectively flow over a layer of air which allows the shear stress to be relieved over the gas layer close to the solid. In simple terms, we can view this as a flow pattern evolving from a Poisieulle flow (Figure 6a) toward a plug flow (Figure 6b), although we are not suggesting full plug flow is achieved. The idea of a small solid area fraction induced kind of superfluidity with pluglike flow occurring in thin channels between two textured parallel plates has also recently been considered theoretically by Feuillebois et al. (36).

The idea of a gas layer to relieve stress and create a large apparent slip length has also previously been suggested by de Gennes, although he related the idea to the existence of gas nanobubbles on the solid surface (37). Vinogradova (38) also considered this problem, and the apparent slip length for a flow of a liquid of viscosity $\eta$ over a gas of viscosity $\eta_{\mathrm{g}}$ and of height $h$ was suggested to be $(33,39)$

$$
b=\left(\frac{\eta}{\eta_{\mathrm{g}}}-1\right) h
$$

For the gas-water interface, the ratio of viscosities is around 50 , and so a $2 \mu \mathrm{m}$ layer of air would be needed for an apparent slip length of the order of $100 \mu \mathrm{m}$, as suggested by the estimates from our low -flow-rate results. This order of magnitude is consistent with the physical structure of the surfaces created in our experiments.

If our observed data are due to the formation of a Cassie state and the effective creation of passively produced aircushioned flow, the removal of slip at higher flow rates could be explained by a transition from the Cassie state to the Wenzel state with increased pressure. The pressure of the transition is below 4 mbar for both the water and the $50 \%$ w/w water-glycerol mixture. Although the viscosity of the water-glycerol mixture is far greater than that of water, the surface tension (40) and contact angle against the surface coating (41) are much the same; thus, the curvature of the interface at any pressure will be similar for the two liquids. From the transition pressure, we can use a simple estimate of the radius of curvature of the water-air interface from $\Delta P=2 \gamma_{\mathrm{LV}} \cos \theta / r$, and this implies a value likely to be greater than $350 \mu \mathrm{m}$, which is too large to match feature size separations of the surfaces. However, recent studies on bubble rafts (42) suggest that, when the pressure increases enough to cause the gas meniscus to curve outward, it behaves as a non-slip surface and the drag induced can even be worse than a flat no-slip surface.

\section{CONCLUSIONS}

In this work we have developed a method to create a superhydrophobic surface on the inside of millimetric diameter copper tubes. The method uses a circulating system that over a long period slowly creates copper hydroxide nanoribbons, which can then be converted to copper oxide. These nanoribbons are easily provided with a hydrophobic coating and thus converted to a superhydrophobic surface. We have developed an experimental system to simultaneously investigate flow rates through four tubes with different internal surface finishes. Our results indicate that, for pressures below around 4 mbar, superhydrophobic tubes have enhanced flow rates and reduced drag for both water and $50 \% \mathrm{w} / \mathrm{w}$ water-glycerol mixtures. The reductions in drag are sufficiently large to suggest that enhanced flow can be directly visualized, and this has been performed using a T-piece test between a superhydrophobic and non-superhydrophobic tube of the same geometrical dimensions. We have interpreted the results as indicating the existence of an effective film of air occurring between the liquid and the solid walls of the tubes which relieves the continuity of stress that would normally result in a no-slip boundary condition for a liquid in direct contact with the solid at all locations along its surface. The principle of using surfaces with superhydrophobic surface finishes to achieve a drag reduction should be applicable to a wide variety of other materials. With further refinement, it should be possible to extend the range of pressures for which a large reduction in drag exists. Materials approaches to increasing drag reduction and robustness of performance could include trading feature size and spacing with apparent slip length, improving the robustness of coatings, and using dual length scale surface features with the larger scale aligned with the direction of flow. In this way, one should be able to create improved tubes and pipes with surfaces designed to retain a film of air without the need for active input of energy and so improve many industrial processes. 
Acknowledgment. We acknowledge financial assistance from the U.K. Engineering and Physical Sciences Research Council (Grant No. EP/D500826/1) and the support of the MOD/Dstl under the Joint Grants Scheme.

Supporting Information Available: Videos showing comparisons of flow through as received and hydrophobic nanoribbon decorated as received tubes. This material is available free of charge via the Internet at http://pubs.acs.org.

\section{REFERENCES AND NOTES}

(1) Leidenfrost, J. G. De aquae communis nonnullis qualitatibus tractatus. University of Duisburg, 1756. (Translation from original Latin: Int. J. Heat Mass Trans., 1966, 9, 1153-1166).

(2) Biance, A. L.; Clanet, C.; Quéré, D. Phys. Fluids 2003, 15, $1632-$ 1637.

(3) Malnig, H. W. ÖIAZ 2001, 146 (3), 96-101.

(4) Cockerell, C. S. Improvements in or relating to vehicles for traveling over land. British Patent 854211, 1960 (filed 1955); equivalent: US Patent 3363716, 1968

(5) McCormick, M. E.; Bhattacharyya, R. Naval Engineers J. 1973, 85, $11-16$.

(6) Kodama, Y. Int. J. Heat Fluid Flow 2000, 21, 582-588.

(7) Thorpe, W. H.; Crisp, D. J. J. Exp. Biol. 1947, 24, 227-269.

(8) Feng, X. L.; Jiang, L. Adv. Mat. 2006, 18, 3063-3078.

(9) Roach, P.; Shirtcliffe, N. J.; Newton, M. I. Soft Matter 2008, 4, 224 240.

(10) Shirtcliffe, N. J.; McHale, G.; Newton, M. I.; Perry, C. C.; Pyatt, F. B. Appl. Phys. Lett. 2006, 89, 104106.

(11) Lauga, E.; Stone, H. A. J. Fluid Mech. 2004, 489, 55-77.

(12) Neto, C.; Evans, D. R.; Bonaccurso, E.; Butt, H.; Craig, V. S. J. Rep. Prog. Phys. 2005, 68, 2859-2897.

(13) Ybert, C.; Barentin, C.; Cottin-Bizonne, C.; Joseph, P.; Bocquet, L. Phys. Fluids 2007, 19, 123601

(14) Watanabe, K.; Udagawa, Y.; Udagawa, H. J. Fluid Mech. 1999, 381, 225-238.

(15) Granick, S.; Zhu, Y. X.; Lee, H. Nature Mat. 2003, 2, 221-227.

(16) Joseph, P.; Cottin-Bizonne, C.; Benoit, J. M.; Ybert, C.; Journet, C.; Tabeling, P.; Bocquet, L. Phys. Rev. Lett. 2006, 97, 156104.

(17) Choi, C. H.; Kim, C. J. Phys. Rev. Lett. 2006, 96, 066001.

(18) Gogte, S.; Vorobieff, P.; Truesdell, R.; Mammoli, A.; van Swol, F.; Shah, P.; Brinker, C. J. Phys. Fluids 2005, 17, 051701.

(19) Yu, Y. S.; Wei, Q. D. Chin. Phys. Lett. 2006, 23, 1634-1637.
(20) Ou, J.; Rothstein, J. P. Phys Fluids 2005, 17, 103606

(21) Cottin-Bizonne, C.; Cross, B.; Steinberger, A.; Charlaix, E. Phys. Rev. Lett. 2005, 94, 056102.

(22) Cottin-Bizonne, C.; Barrat, J. L.; Bocquet, L.; Charlaix, E. Nature Mat. 2003, 2, 237-240.

(23) Bocquet, L.; Tabeling, P.; Manneville, S. Phys. Rev. Lett. 2006, 97 , 109601.

(24) Lee, C.; Choi, C. H.; Kim, C. J. Phys. Rev. Lett. 2008, 101, 064501.

(25) McHale, G.; Shirtcliffe, N. J.; Evans, C. R.; Newton, M. I. Appl. Phys. Lett. 2009, 94, 064104.

(26) Landau, L. D.; Lifshitz, E. M. Fluid Mechanics; Pergamon Press: London, 1959

(27) Hadamard, J. S. C. R. Hebd. Seances Acad. Sci. (Paris) 1911, 152, 1735-1738.

(28) De Rybczynski, M. W. Bull. Acad. Sci. Cracovie Ser. A 1911, 40 46.

(29) Boussinesq, J. C. C. R. Hebd. Seances Acad. Sci. (Paris) 1913, 156, 983-989.

(30) Wen, X. G.; Zhang, W. X.; Yang, S. H. Langmuir 2003, 19, 5898 5903.

(31) Léger, L.; Hervet, H.; Massey, G.; Durliat, E. J. Phys. Cond. Matt. 1997, 9, 7719-7740.

(32) McHale, G.; Lücklum, R.; Newton, M. I.; Cowen, J. A. J. Appl. Phys. 2008, 88, 7304-7312.

(33) Vinogradova, O. I. Int. J. Mineral Proc. 1999, 56, 31-60.

(34) Neto, C.; Evans, D. R.; Bonaccurso, E.; Butt, H.; Craig, V. S. J. Rep. Prog. Phys. 2005, 68, 2859-2897.

(35) Shirtcliffe, N. J.; McHale, G.; Newton, M. I.; Chabrol, G.; Perry, C. C. Adv. Mater. 2004, 16, 1929-1932.

(36) Feuillebois, F.; Bazant, M. Z.; Vinogradova, O. I. Phys. Rev. Lett 2009, 102, 026001.

(37) de Gennes, P. G. Langmuir 2002, 18, 3413-3414.

(38) Vinogradova, O. I. Langmuir 1995, 11, 2213-2220.

(39) Lauga, E., Brenner, M. P., Stone, H. A., Microfluidics: the no-slip boundary condition. In Handbook of Experimental Fluid Dynamics; Springer: New York, 2006.

(40) Khossravi, D.; Connors, K. A. J. Solution Chem. 1993, 22, 321 330 .

(41) Roach, P.; McHale, G.; Evans, C. R.; Shirtcliffe, N. J.; Newton, M. I. Langmuir 2007, 23, 9823-9830.

(42) Steinberger, A.; Cottin-Bizonne, C.; Kleimann, P.; Charlaix, E. Nature Mat. 2007, 6, 665-668.

AM9001937 